\title{
A Convenient Total Synthesis of (+)-Decursinol from Resorcinol
}

\author{
Jung Ho Lee, Hyun Bae Bang, Su Young Han, and Jong-Gab Jun* \\ Deparment of Chemistr, Institute of Applied Chemistry, Hallw Cniversin, Chunchon 200-702. Korea \\ "E-mail: jginiá hallymackr \\ Received September 27, 2006
}

Key Words : Decursinol. Resorcinol, Jacobsen's epoxidation, Xanthỵletin

(+)-Decursinol (1), a class of linear dihỵdrocoumarin natural product was isolated from the root of Angelica gigas Nakai (Umbelliferae) ${ }^{l}$ which is a traditional medicine for anemia in Korea. - It has attracted increasing attention due to its prominent biological activities. It exhibits cytotoxic activity related with protein kinase $\mathrm{C}$ (PKC) against several human cancer cell line. ${ }^{3}$ anti-helicobacter pylori activity. ${ }^{4}$ and strong antinociceptive activity: The asymetric total syntheses of 1 were reported by Shibasaki ${ }^{6} \operatorname{Han}^{7}$ and $\mathrm{Kim}^{8}{ }^{8}$ The Shibasaki, the Han and the Kim groups used esculetin (2). resorcinol (3). and umbelliferone (4) respectively as a starting material for (+)-decursinol șynthesis. This work focused on the resorcinol as a starting material. since it was cheaper and simpler material. and reported herein is an efficient enantioselective total synthesis of (+)-decursinol in five steps from resorcinol.

The Han group syinthesized (+)-decursinol from resorcinol in 7 steps as shown in Scheme 1. The serious problem in their synthesis is the final condensation step which produced equimolar amount of regioisomer (11) resulting in a low yield of desired product 1. Asymumetric epoxidation of 6 to 7. which requires an expensive Jacobsen's (salen)-Mn catalyst. ${ }^{9}$ is the key step for introducing desired chirality on 7 -hydrosyl group in product 1 . A condensation reaction of ethyl propiolate with the compounds 5, 6. and 7 respectively was done before epoxidation step for economical reason. but it failed. The condensation of phenol derivatives with ethyl propiolate is very sensitive to the functionality (conjugation) of substituents. for instance. 10 reacts but 6 doesn't. Umbelliferone (4) also does not react with ethyl propiolate or 3methyl-2-butenoic acid in this condensation reaction.

This work proposes herein a convenient and practical total synthesis of $(+)$-decursinol from resorcinol in five steps. The commercially available resorcinol (3) is condensed with 2methyl-3-buten-2-ol using zinc chloride under reflux to give 12 in $70 \%$ yield as shown in Scheme 2 (entry 2). Acid catalysts (entries 1,9) or acid solvents (entries 6. 7) also gave a reasonable yield in this reaction. The subsequent reaction with ethyl propiolate only using zinc chloride produced the $1: 1$ mixture of corresponding lactones 13 and 14. which is easily separated by column chromatography in $38 \%$ yields respectively (Scheme 3). The fomation of regio-

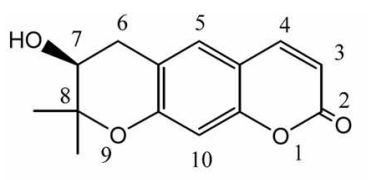

1 (+)-decursinol<smiles>Oc1cccc(O)c1</smiles>

3 resorcinol<smiles>O=c1ccc2cc(O)c(O)cc2o1</smiles>

2 esculetin<smiles>O=c1ccc2ccc(O)cc2o1</smiles>

4 umbelliferone
Figure 1. Structure of (+)-decursinol, esculetin, resorcinol and umbelliterone.<smiles>CC(C)(C)C1(C)CC(=O)c2ccc(O)cc2O1</smiles>

Scheme 1. Han's synthesis: (a) 3-methyl-2-butenoic acid, $\mathrm{MeSO}_{3} \mathrm{H}, \mathrm{P}_{2} \mathrm{O}_{5}, 70{ }^{\circ} \mathrm{C}, 96 \%$, (b) LAH, THF, reflux, $83 \%$; (c) $p$-TsOH, THF, reflux, $88 \%$; (d) acetic anlydride, pyidine, DMAP, $\mathrm{CH}_{2} \mathrm{Cl}_{2}$, rt, 98\%; (e) Jacobsen's ( $S, S$ )-salen-Mn(III) catalyst, $n$-BunNHSO, buttered solution/ $\mathrm{CH}_{3} \mathrm{CN}, 1,1,1$-tritluoroacetone, Oxone ${ }^{\mathrm{E}}, \mathrm{NaHCO}_{3}, 0^{\circ} \mathrm{C}, 83 \%$, (f) LAH, THF, $0^{\circ} \mathrm{C}, 81 \%$; (g) ethyl propiolate, zinc chloride, $110^{\circ} \mathrm{C}$, $40 \%$ 


\begin{tabular}{|c|c|c|c|c|c|}
\hline entry & acid & eq $(\mathrm{mol})$ & solvent & time(h) & Yield $(\%)^{\circ}$ \\
\hline 1 & p-TsOH & 0.1 & dichloromethane & 6 & 65 \\
\hline 2 & $\mathrm{ZnCl}_{2}$ & 1 & dichloromethane & 7 & 70 \\
\hline 3 & $\mathrm{H}_{2} \mathrm{SO}_{4}$ & Cat. & dichloromethane & 4 & 37 \\
\hline 4 & $\mathrm{AlCl}_{3}$ & 1 & dichloromethane & 7 & * \\
\hline 5 & $\mathrm{BF}_{3} \mathrm{Et}_{2} \mathrm{O} / \mathrm{TMSOMS}$ & 0.110 .5 & dichloromethane & 7 & 20 \\
\hline 6 & $\mathrm{HCO}_{2} \mathrm{H}$ & solvenl & & 4 & 64 \\
\hline 7 & $\mathrm{AcOH}$ & solvent & & 4 & 63 \\
\hline 8 & $\mathrm{MeSO}_{3} \mathrm{H}$ & solvent & & 7 & , \\
\hline 9 & $\mathrm{MeSO}_{3} \mathrm{H}$ & Cat & dichloromethane & 6 & 58 \\
\hline
\end{tabular}

Scheme 2. Condensation reaction of resorcinol with 2-methyl-3butene-2-ol.

\begin{tabular}{llllllll}
\hline & & & & & & \\
\hline
\end{tabular}

*reaction temp. $\left(110^{\circ} \mathrm{C}\right){ }^{* *}$ isolated yield

Scheme 3 . Condensation reaction of 12 with ethyl propiolate.

isomer $\mathbf{1 4}$ could not be minimized in several different reaction conditions. Despite the low yield of desired lactone 13. the Han 's problem ${ }^{7}$ could be overcome by introducing this troublous step at an early stage before chiral epoxidation rather than at a final stage.

The lactone $\mathbf{1 3}$ is dehydrogenated by DDQ in refluxing benzene to give xanthyletin (15) in $82 \%$ yield (Scheme 4$)^{\text {ID }}$ The alternative use of NBS/AIBN in refluxing $\mathrm{CCl}_{4}$ produced a low yield of 15. Xanthyletin have been isolated from tissues of Citrus infected by Phytophthora spp. and known as an efficient growth inhibitor of Phytophthora citrophthora. ${ }^{11}$ The xanthyletin 15 was converted to the desired chiral epoxide 16 in $83 \%$ yield $(95 \%$ ee) in the presence of Jacobsen`s $(S, S)-(+)$-salen-Mn(III) catalyst with Oxone ${ }^{\mathbb{E}}$ using the procedure developed by Han. ${ }^{\text {p.y }}$ The absolute configuration of the epoxide 16 was deternined by its transformation to the authentic natural $(+)$-decursinol 1 . The

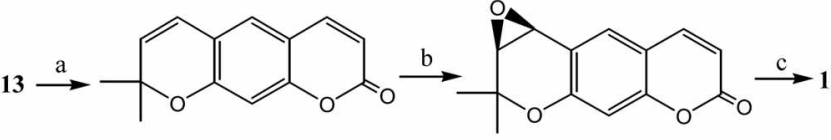

15

16

Scheme 4. Reagents and conditions: (a) DDQ $\mathrm{PlH}$, reflux, $82 \%$; (b) Jacobsen`s $(S, S)$-Salen-Mn(III) catalyst, $n$-Bu+NHSO, buffered solution $/ \mathrm{CH}_{3} \mathrm{CN}, 1,1,1$-trifluoroacetone, Oxone ${ }^{\mathrm{i}}, \mathrm{NaHCO}_{3}, 0{ }^{\circ} \mathrm{C}$, $83 \%$; (c) $\mathrm{NaBH}_{3} \mathrm{CN}_{2}, \mathrm{BF}_{3} \mathrm{OEt}_{2}, \mathrm{THF}, \mathrm{rt}, 93 \%$.

regio- and stereoselective reduction of 16 by using $\mathrm{NaBH}_{3} \mathrm{CN}$ with $\mathrm{BF}_{3} \cdot \mathrm{OEt}_{2}$ in THF at rt gave (+)-decursinol in $93 \%$ yield. ${ }^{8}$

In conclusion, the enantioselective and convenient synthesis of $(+)$-decursinol has been achieved from resorcinol in five steps with $16.8 \%$ overall yield including double condensation. oxidation. chiral epoxidation, and reduction. The introduction of ethyl propiolate condensation step. which produced equimolar amount of regioisomer resulting in low yield of 1 . at an early stage lead to a practical and economical synthesis of $(+)$-decursinol.

\section{Experimental Section}

All chemicals used were purchased from commercial sources and used as received unless otherwise stated. NMR spectra were recorded at Varian Gemini-300 MHz FT-NMR for ${ }^{1} \mathrm{H}$ and $75.5 \mathrm{MHz}$ for ${ }^{13} \mathrm{C}$, with the chemical shifts $(\delta)$ reported in parts per million (ppm) relative to TMS and the coupling constants $(J)$ quoted in $\mathrm{Hz}$. $\mathrm{CDCl}_{3}$ was used as a solvent and an internal standard. Flash chromatography was carried out using silica gel Merck 60 (230-400 mesh). Thinlayer chromatography (TLC) was perfomed on DC-Plastikfolien $60 . \mathrm{F}_{254}$ (Merck, layer thickness $0.2 \mathrm{~mm}$ ) plasticbacked silica gel plates with visualization by UV light (254 $\mathrm{nm}$ ) or by treatment with $p$-anisaldehyde. Optical rotation was determined on a Rudolph Research AUTOPOL ${ }^{1}$ III polarimeter with $10 \mathrm{~cm}$ tube. Melting points were measured on a MEL-TEMP II apparatus and were uncorrected.

7-Hydroxy-2,2-dimethylchroman (12). To a solution of resorcinol 3 (1.00 g. $9.08 \mathrm{~mm}$ ol) in dichloromethane (20 $\mathrm{mL}$ ) was added zinc chloride $(0.62 \mathrm{~g} .4 .54 \mathrm{mmol})$ followed by 2-methyl-3-buten-2-ol $(0.48 \mathrm{~mL} .4 .54 \mathrm{mmol})$ and stirred for $2 \mathrm{~h}$ at $\mathrm{rt}$. The reaction mixture was heated to reflux for 7 $\mathrm{h}$. After being cooled. the mixture was neutralized with saturated $\mathrm{NaHCO}_{3}$ and extracted with dietllyl ether. Concentration and column cluromatography (EtOAc : Hexane $=1$ : 9) gave the white solid $8(0.55 \mathrm{~g} .70 \%) \cdot R_{\mathrm{f}} 0.60$ (EtOAc : Hexane $=1: 2) ; \mathrm{mp} 62-64{ }^{\circ} \mathrm{C}:{ }^{1} \mathrm{H}$ NMR $\left(300 \mathrm{MHz} . \mathrm{CDCl}_{3}\right)$ $\delta 1.31(6 \mathrm{H}, \mathrm{s} . \mathrm{C} 2-\mathrm{methyls}) .1 .77(2 \mathrm{H} . \mathrm{t} . J=6.6 \mathrm{~Hz} . \mathrm{C} 3-\mathrm{H})$, $2.68(2 \mathrm{H} . \mathrm{t}, J=6.6 \mathrm{~Hz}, \mathrm{C} 4-\mathrm{H}), 4.97(\mathrm{lH} . \mathrm{s} . \mathrm{OH}), 6.27(\mathrm{lH}$, br s. C8-H), $6.32(1 \mathrm{H}$. br d. $J=8.1 \mathrm{~Hz}$. C6-H). $6.88(1 \mathrm{H} . \mathrm{d} . J$ $=8.1 \mathrm{~Hz}, \mathrm{C} 5-\mathrm{H}) \cdot{ }^{13} \mathrm{C}$ NMR $\left(75 \mathrm{MHz}, \mathrm{CDCl}_{3}\right) \delta 22.1(\mathrm{C} 3)$. $27.2(2 \mathrm{Me}), 33.3(\mathrm{C} 4), 74.6(\mathrm{C} 2), 104.0(\mathrm{C} 8) .107 .5(\mathrm{C} 6)$. 113.5 (C4a). 130.2 (C5). 154.9 (C7). 155.0 (C8a).

8,8-Dimethyl-7,8-dihydro-6H-pyrano[3,2-g] chromen-2one (13) and 6,6-dimethyl-6,7-dihydro-8H-pyrano[2,3-f]chromen-2-one (14). To a chroman $12(0.55 \mathrm{~g} .3 .08 \mathrm{mmol})$ 
was added ethyl propiolate $(0.47 \mathrm{~mL}, 4.62 \mathrm{mmol})$ with zinc chloride $(0.41 \mathrm{~g} .3 .08 \mathrm{mmol})$. and heated at $110^{\circ} \mathrm{C}$ for $1.5 \mathrm{~h}$. After being cooled the reaction was quenched with $5 \%$ aqueous $\mathrm{HCl}$ and extracted with EtOAc. Concentration and column chromatograply (EtOAc: Hexane $=1: 7$ ) gave the white solid $13(0.27$ g. $38 \%)$ and $14(0.27$ g. $38 \%)$. $13: R_{\mathrm{f}}$ $0.68($ EtOAc : Hexane $=1: 2): \mathrm{mp} 102-104{ }^{\circ} \mathrm{C}:{ }^{1} \mathrm{H}$ NMR (300 MHz. CDCl $) \delta 1.36$ (6H. s. C8-methyls), 1.84 (2H. t. $J$ $=6.6 \mathrm{~Hz}, \mathrm{C} 7-\mathrm{H}), 2.82(2 \mathrm{H}, \mathrm{t} . J=6.6 \mathrm{~Hz}, \mathrm{C} 6-\mathrm{H}) .6 .18(1 \mathrm{H}, \mathrm{d}$. $J=9.3 \mathrm{~Hz} . \mathrm{C} 3-\mathrm{H}) .6 .70(1 \mathrm{H}$. s. Cl0-H) 7.14 (1H. s. C $5-\mathrm{H})$. $7.56(1 \mathrm{H} . \mathrm{d} . J=9.3 \mathrm{~Hz} . \mathrm{C} 4-\mathrm{H}) .{ }^{13} \mathrm{C}$ NMR $\left(75 \mathrm{MHz} . \mathrm{CDCl}_{3}\right)$ $\delta 22.2(\mathrm{C} 7), 27.2(2 \mathrm{Me}) .32 .7(\mathrm{C} 6), 76.0(\mathrm{C} 8) .104 .8(\mathrm{C} 3)$. 112.4 (C4a). 112.9 (C10), 118.6 (C5a). 128.4 (C5) 143.4 (C4). 154.1 (C10a) 157.8 (C9a). 161.7 (C2). 14: $R_{\mathrm{f}} 0.81$ $($ EtOAc : Hexane $=1: 2): m p 172-175^{\circ} \mathrm{C}:{ }^{1} \mathrm{H}$ NMR $(300$ MHz. $\left.\mathrm{CDCl}_{3}\right) \delta 1.38(6 \mathrm{H}, \mathrm{s}$. C6-methyls), $1.84(2 \mathrm{H}, \mathrm{t}, J=$ $6.6 \mathrm{~Hz}, \mathrm{C} 7-\mathrm{H}) .2 .77(2 \mathrm{H}, \mathrm{t} J=6.6 \mathrm{~Hz} . \mathrm{C} 8 \mathrm{-H}) .6 .28(1 \mathrm{H} . \mathrm{d} . J$ $=9.6 \mathrm{~Hz}, \mathrm{C} 3-\mathrm{H}) .6 .77(1 \mathrm{H}, \mathrm{d} . J=8.5 \mathrm{~Hz}, \mathrm{Cl}-\mathrm{H}) .7 .17(1 \mathrm{H}$. d. $J=8.5 \mathrm{~Hz} . \mathrm{C} 9-\mathrm{H}) .8 .06(1 \mathrm{H} . \mathrm{d} . J=9.6 \mathrm{~Hz} \cdot \mathrm{C} 4-\mathrm{H}) .{ }^{13} \mathrm{C}$ $\operatorname{NMR}\left(75 \mathrm{MHz} . \mathrm{CDCl}_{3}\right) \delta 22.2(\mathrm{C} 7), 27.2(2 \mathrm{Me}), 32.7(\mathrm{C} 8)$. 76.1 (C6). 107.8 (C3). 109.5 (C4a). 114.1 (C10). 115.9 (C8a). 132.8 (C9). 139.0 (C4). 150.4 (C10a). 153.7 (C4b). $161.5(\mathrm{C} 2)$.

Xanthyletin (15). To a solution of chromenone $13(0.16 \mathrm{~g}$. $0.69 \mathrm{mmol})$ in benzene $(30 \mathrm{~mL})$ was added DDQ $(0.37 \mathrm{~g}$. $2.06 \mathrm{mmol}$ ) and refluxed for $40 \mathrm{~h}$. After being cooled. the reaction was quenched with saturated aqueous $\mathrm{NaHSO}_{3}$ and extracted with diethyl ether. Concentration and column chromatography (EtOAc : Hexane $=1: 4$ ) gave the pale yellow solid $15(0.13 \mathrm{~g} .82 \%) . R_{\mathrm{f}} 0.73$ (EtOAc : Hexane $=1$ : 2): $\mathrm{mp} 88-90{ }^{\circ} \mathrm{C}:{ }^{1} \mathrm{H} \mathrm{NMR}\left(300 \mathrm{MHz}, \mathrm{CDCl}_{3}\right) \delta 1.47(6 \mathrm{H}$. s. C8-methyls) $5.68(2 \mathrm{H}$ d. $J=9.9 \mathrm{~Hz}$. C6-H). 6.21 (1H. d. $J=9.6 \mathrm{~Hz} . \mathrm{C} 3-\mathrm{H}) .6 .33(1 \mathrm{H} . \mathrm{d} . J=9.9 \mathrm{~Hz} . \mathrm{C} 7 \mathrm{-H}) .6 .71(1 \mathrm{H}$. s. Cl0-H). $7.03(1 \mathrm{H}$ s. C5-H). $7.56(1 \mathrm{H}$. d. $J=9.3 \mathrm{~Hz} . \mathrm{C} 4-$ H). ${ }^{13} \mathrm{C}$ NMR $\left(75 \mathrm{MHz}, \mathrm{CDCl}_{3}\right) \delta 28.7(2 \mathrm{Me}) .77 .9(\mathrm{C} 8)$. 104.6 (C3). 112.9 (C4a). 113.2 (C10). 118.6 (C5a). 120.9 (C6). 124.9 (C7). 131.3 (C5) 143.4 (C4). 155.5 (C10a). 156.9 (C9a). 161.2(C2).

(6S,7R)-6,7-Epoxy-8,8-dimethyl-7,8-dihydro-6 $H$-pyrano[3,2-g]chromen-2-one (16). To a solution of xanthyletin 15 $(0.10 \mathrm{~g} .0 .44 \mathrm{mmol})$ in $\mathrm{CH}_{3} \mathrm{CN}(5 \mathrm{~mL})$ at rt was added $(S, S)$ (+)-N:N'-bis(3.5-di-t-butylsalicylidene)-1.2-cy'clohexanediaminomanganese(II) chloride (Jacobsen's (S,S)-salen$\mathrm{Mn}$ (III) catalyst. $5 \mathrm{mg} .0 .018 \mathrm{mmol}$ ) and $n-\mathrm{Bu}_{4} \mathrm{NHSO}_{4}(5.3$ mg. $0.015 \mathrm{mmol}$ ) with a buffer solution of $50 \mathrm{mM} \mathrm{Na}=\mathrm{B}_{4} \mathrm{O}_{7}$. $10 \mathrm{H}_{2} \mathrm{O}$ in $0.4 \mathrm{mM}$ aqueous $\mathrm{Na}_{3}$ EDTA $(3.5 \mathrm{~mL})$. After the reaction mixture was cooled to $0^{\circ} \mathrm{C}$. 1,1.1-trifluoroacetone $(0.05 \mathrm{~mL})$ was added. followed by portionwise addition of two solutions of Oxone ${ }^{(\mathrm{p})}(1.0 \mathrm{~g}, 1.62 \mathrm{mmol})$ in $0.4 \mathrm{mM}$ aqueous $\mathrm{Na}_{2}$ EDTA $(5 \mathrm{~mL})$ and $\mathrm{NaHCO}_{3}(0.3 \mathrm{~g} .3 .57 \mathrm{~nm}$ in $\mathrm{H}_{3} \mathrm{O}(5 \mathrm{~mL})$ with stirring over the reaction period $(1.5 \mathrm{~h})$.

The reaction mixture was then treated with water and extracted with diethyl ether. Concentration and column chromatography (EtOAc: hexane $=1: 2$ ) gave the white solid 16 (85 mg. 83\%). $R_{\mathrm{f}} 0.35($ EtOAc : Hexane $=1: 2)$; $\mathrm{mp}$ $135-140{ }^{\circ} \mathrm{C} ;{ }^{1} \mathrm{H}$ NMR $\left(300 \mathrm{MHz}_{2} \mathrm{CDCl}_{\hat{j}}\right) \delta 1.3 \mathrm{l}(3 \mathrm{H} . \mathrm{s}, \mathrm{Me})$.
1.61 (3H. s. Me). 3.54 (1H. d, $J=4.5 \mathrm{~Hz}, \mathrm{C} 7-\mathrm{H}), 3.97(\mathrm{HH}$. d. $J=4.5 \mathrm{~Hz}, \mathrm{C} 6-\mathrm{H}) .6 .26(\mathrm{lH} . \mathrm{d} . J=9.6 \mathrm{~Hz}, \mathrm{C} 3-\mathrm{H}), 6.76$ $(1 \mathrm{H}$. s. Cl0-H). $7.45(1 \mathrm{H}$. s. C $5-\mathrm{H}) .7 .62(1 \mathrm{H} . \mathrm{d} . J=9.6 \mathrm{~Hz}$. C4-H). ${ }^{13} \mathrm{C} \mathrm{NMR}\left(75 \mathrm{MHz} . \mathrm{CDCl}_{3}\right) \delta 23.5(\mathrm{Me}), 25.8(\mathrm{Me})$. 50.5 (C7). 62.3 (C6). 74.8 (C8). 106.3 (C3). 113.3 (C+a). 114.1 (C.10), 117.5 (C5a), 129.0 (C5), 143.0 (C4). 156.2 (C9a \& C10a). 1609 (C2).

(+)-Decursinol (1). To a solution of epoxide $16(0.035 \mathrm{~g}$. $0.143 \mathrm{mmol})$ in THF $(10 \mathrm{~mL})$ was added $\mathrm{NaBH}_{3} \mathrm{CN}(0.011$ g. $0.172 \mathrm{mmol})$ with $\mathrm{BF}_{3} \cdot \mathrm{OEt}_{2}(0.018 \mathrm{~g} .0 .143 \mathrm{mmol})$ at $0^{\circ} \mathrm{C}$ and stirred at $\mathrm{rt}$ for $0.5 \mathrm{~h}$. The reaction was quenched with saturated aqueous $\mathrm{NaHCO}_{3}$ and extracted with EtOAc Concentration and colunun chromatograply (EtOAc: Hexane $=1: 2)$ gave the white solid $1(0.033$ g. $93 \%) . R_{f} 0.13$ (EtOAc: Hexane $=1: 2): \operatorname{mp~} 167-170^{\circ} \mathrm{C} ;[\alpha]_{\mathrm{D}}^{26}=+10.3(\mathrm{c}$ 1.0. $\mathrm{CHCl}_{3} .95 \%$ ee) (lit. $\left.{ }^{12}[\alpha]_{\mathrm{D}}^{26}=+10.8\right)$ : ${ }^{1} \mathrm{H}$ NMR $(300$ $\left.\mathrm{MHz}, \mathrm{CDCl}_{3}\right) \delta 1.36$ (3H. s, Me). 1.39 (3H, s, Me). 2.00 $(1 \mathrm{H}, \mathrm{br} \mathrm{s} . \mathrm{OH}) .2 .83(1 \mathrm{H} . \mathrm{dd} . J=5.7,16.5 \mathrm{~Hz}, \mathrm{C} 6-\mathrm{H}) .3 .10$ (1H. dd $J=5.7,16.5 \mathrm{~Hz}, \mathrm{C} 6-\mathrm{H}) .3 .86(1 \mathrm{H}$, br t. $J=5.4 \mathrm{~Hz}$. C7-H). $6.19(1 \mathrm{H}, \mathrm{d} . J=9.3 \mathrm{~Hz}, \mathrm{C} 3-\mathrm{H}) .6 .76(1 \mathrm{H}, \mathrm{s} . \mathrm{ClO}-\mathrm{H})$. $7.16(1 \mathrm{H}$. s. C5-H). 7.56 (1H. d. $J=9.3 \mathrm{~Hz} . \mathrm{C} 4-\mathrm{H}) .{ }^{12} \mathrm{C}$ NMR (75 MHz. $\left.\mathrm{CDCl}_{3}\right) \delta 22.4(\mathrm{Me}), 25.4(\mathrm{Me}) .31 .0(\mathrm{C} 6)$, 69.3 (C7). 78.5 (C8). 104.9 (C3). 113.1 (C4a). 113.3 (Cl0). 116.8 (C5a). 129.2 (C7). 143.4 (C6), 154.2 (C.10a), 156.7 (C9a). 161.6 (C2).

Acknowledgements. This work was supported by grant No. (R01-2005-000-10916-0) from the Basic Research Program of Korea Science \& Engineering Foundation. and in part by the Ministry of Commerce. Industry and Energy through the Center for Efficacy Assessment and Development of Functional Foods and Drugs at Hallym University. Korea.

\section{References}

1. Hata. K.: Sano, K. Tetrahedron Lett. 1966. 7, 1461-1465.

2. Han. D. S. Phamnacogiosy. $4^{\text {th }}$ ed.: Dongmyongsa: Seoul, 1992.

3. Ahn. K.-S.; Sim. W.-S.: Kim. I.-H. Planta lled 1996, 62, 7-9

4. Bae. E.-A.: Han. M. J.: Kim. N.-J.: Kim. D.-H. Bio. Pham Bull. 1998. 21.990-992.

5. Choi. S.-S.: Han. K-T.: Lee. T-K.: Lee. H.-K.: Han. E--T.: Kim. D.-H.: Suh. H. W. Life Sciences 2003. 73. 471-485.

6. Nemoto. T.: Ohshima. T.: Shibasaki. M. Tetrohedhon Lett. $\mathbf{2 0 0 0 .}$ H. $9569-9574$.

7. Lim. T.: Kiml. I.-H.: Kimn. H. H.: Ahn. K.-S.: Han. H. Tetrohedon Lett. 2001 , 2. 4001-4003.

8. Kim. S.; Ko. H.; Son. S.; Shin. K. J.; Kim. D. J. Tetrahedron Lett. 2001. 42,7641-7643

9. Jacobsen. E. N.; Zhang, W.; Muci. A. R.; Ecker. J. R.; Deng, L. J. Am. Chem Soc. 1991, 113, 7063-7064.

10. (a) Steelink. C.: Marshall, G. P. J. Org. Chem. 1979. if, 14291433. (b) Lee, K. Y.; Kim. S. C.; Kim. J. N. Bull. Kowan Chem. Soc. 2005, 26, 2078-2080.

11. Khan. A. J.: Kunesch. G.: Chuilon. S.: Ravise, A. Fruits 1985, 40. $807-811$.

12. (a) Lemmich, J.: Nielsen. E. Tetrahedron Lett. 1969, 10, 3-4. (b) Nemoto. T.: Ohshima, T; Shibasaki, M. Tetrahedron 2003, 59. 6889-6897. 